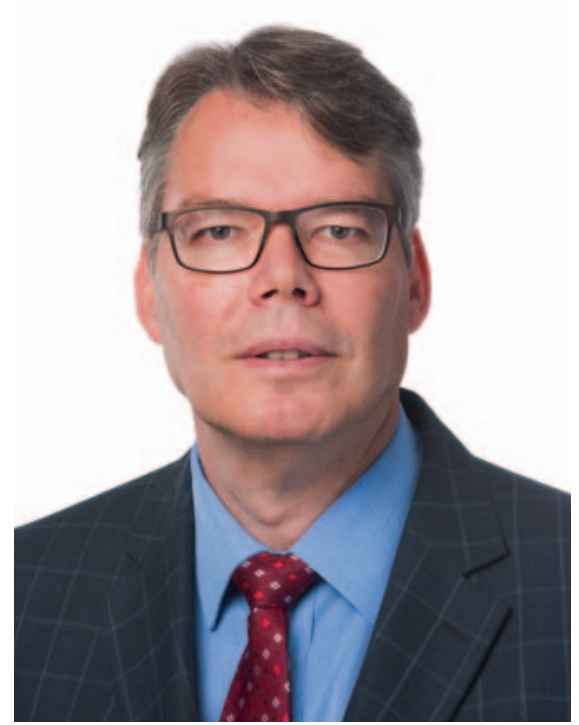

\section{Sie sind bei Swissmedic ein "alter Hase" und mit der Abteilung KPA bereits bestens vertraut. Um Sie besser kennenzulernen: Was gibt es zur Person Dr. Martin Ziak zu berichten?}

Aufgewachsen bin ich in Winterthur. Ich habe an der Universität Zürich Biochemie studiert, promoviert und dann 20 Jahre lang als Forschungsgruppenleiter in der medizinisch-biologischen Grundlagenforschung gearbeitet. Nebst der Forschungstätigkeit war ich Lehrbeauftragter an der Universität Zürich. 2009 wechselte ich zur Swissmedic. Dort betreute ich als Leiter der Einheit 4 des Case Managements bereits KPA. Als Ausgleich zum Beruf bin ich gerne im Garten. Auch dort habe ich mit Pflanzen und Kräutern zu tun.

\title{
Die Abteilung Komplementär- und Phytoarzneimittel der Swissmedic unter neuer Leitung
}

Die Anforderungen für die Zulassung von Komplementär- und Phytoarzneimitteln (KPA) geben immer wieder Anlass zu Diskussionen. Die Abteilung, die bei Swissmedic darüber entscheidet, hat jetzt einen neuen Leiter. Es ist der Biochemiker Dr. Martin Ziak. Sein Ziel: Die Zulassung dieser Heilmittel soll noch einfacher werden, damit die Vielfalt des Angebots für die Patienten erhalten bleibt. Für die Zukunft der KPA sieht er ein grosses Potenzial.

\section{Welche Unterschiede bzw. zusätzlichen Aufgaben sehen Sie im Vergleich zu Ihrer bisherigen Position als Einheitsleiter im Case Management bei Swissmedic? Wie sehen Ihre Ziele aus?}

Als Abteilungsleiter bin ich stärker in strategische Entscheide des Bereichs Zulassung involviert, d.h., ich kann mitgestalten und die Interessen für KPA einbringen. Ferner besitze ich als Abteilungsleiter auch mehr Kompetenzen. Ich übernehme eine TriageFunktion und definiere die Verantwortlichkeiten für die Begutachtung von KPA-Präparaten.

Nach der Volksabstimmung von 2009 sagte das Schweizer Volk klar und deutlich, dass es der Komplementärmedizin einen hohen Stellenwert einräumt. KPA geniessen in der Bevölkerung eine breite Akzeptanz. Ich will mit meinem Team sicherstellen, dass eine breite Palette dieser Arzneimittel zur Verfügung steht. Mein Ziel ist es aber auch, dass die Vielfalt dieser Arz- neimittel erhalten bleibt. Das heisst, die Patientinnen und Patienten sollen die Wahl haben. Dazu ist es wichtig, dass die Zulassung für KPA noch einfacher wird. Für pflanzliche Arzneimittel bedeutet dies, dass die Wirksamkeit und Sicherheit durch einen bibliographischen Nachweis erfolgen kann. Komplementär-Arzneimittel sollen entsprechend der Therapierichtung beurteilt werden. Das heisst: Wenn es um die Zulassung eines homöopathischen Heilmittels geht, dann wird ein Homöopathie-Experte beigezogen. Die Anforderung, dass nur qualitativ einwandfreie, sichere und aus der Sicht der jeweiligen Fachexperten wirksame Arzneimittel zugelassen und in den Verkehr gebracht werden dürfen, bleibt selbstverständlich bestehen.

Aus welchem Anlass wurde die Abteilung KPA umorganisiert?

Das Heilmittelgesetz (HMG) wird im Moment überarbeitet. Darin sind 
zusätzliche Erleichterungen beim $\mathrm{Zu}$ lassungsverfahren für Arzneimittel der Komplementärmedizin und Pflanzenheilkunde vorgesehen, und darauf wollen wir uns vorbereiten.

\section{Wie sieht denn die Situation der KPA im Rahmen der Revision des HMG aus?}

Zum jetzigen Zeitpunkt ist es noch zu früh, um die konkreten Auswirkungen der Revision auf KPA abzuschätzen. Mit den jetzigen Vereinfachungen bei der Zulassung haben wir bereits eine gute Grundlage. Wir nehmen aber das Signal auf, die regulatorische Praxis zu diesen Arzneimitteln zukünftig noch mehr zu vereinfachen.

\section{Was sind die nächsten Massnahmen?}

Es ist geplant, Evaluationsberichte öffentlich zu machen, d.h., wir machen öffentlich, warum ein Heilmittel zugelassen wurde oder nicht. Zuerst für alle beteiligten Parteien, später für alle Interessierten. Die Begutachtungspraxis von Swissmedic wird damit nachvollziehbar und transparent. Das ist uns wichtig, um den Mentalitätswechsel zu signalisieren, der mit der konsequenten Umsetzung des Willens der Bevölkerung bezüglich Komplementärmedizin verbunden ist.

\section{Was sind Ihre bisherigen Berührungspunkte mit der Komplementärmedizin?}

Falls ich einmal Patient bin, schätze ich es, dass es nebst synthetischen Arzneimitteln auch KPA-Präparate gibt. Ich nehme dann auch einmal einen Tee oder ein Arzneimittel, welches pflanzliche Wirkstoffe enthält.
Wie wichtig ist lhrer Ansicht nach die Entwicklung neuer Medikamente in der Komplementärmedizin insbesondere in der Schweiz? Welchen Beitrag kann Ihre Abteilung hierzu leisten?

In den letzten Jahren wurden immer weniger KPA zur Zulassung angemeldet. Das ist äusserst schade, denn wir brauchen die Vielfalt dieser Präparate. Für Kleinunternehmen sind klinische Studien oft zu teuer und zeitintensiv. Deshalb beantragen die Zulassungsinhaber immer öfter eine traditionelle Anwendung von Heilmitteln und beziehen sich auf publizierte Literaturdaten oder Monographien. Generell muss das Zulassungsverfahren weiter vereinfacht werden und die Begutachtung pragmatisch sowie risikobasiert unter Berücksichtigung der gesetzlichen Vorgaben erfolgen. Bei den Zulassungsgebühren profitieren die KPA-Präparate schon heute von Gebührenreduktionen.

\section{Welche Rolle spielt dabei Ihrer Meinung nach die Europäische Union mit ihren Vorschriften?}

Grundsätzlich hat die Schweiz ihre eigenen Gesetze - und die sind für uns entscheidend. Die Zulassung von KPA ist in der Schweiz in vielfacher Hinsicht anders geregelt als in der Europäischen Union. In der schweizerischen Gesetzgebung fehlt beispielsweise die Unterscheidung zwischen traditionellen pflanzlichen Arzneimitteln und den Arzneimitteln mit «wellestablished medicinal use» (d.h. allgemein medizinisch anerkannte). Ferner lässt die europäische Gesetzgebung bei traditionellen pflanzlichen Arzneimitteln auch Kombinationen von pflanzlichen Bestandteilen mit Vitaminen und Mineralstoffen zu. Der Begriff der traditionellen pflanzlichen Arzneimit- tel ist somit nicht deckungsgleich. Zusammengefasst kann gesagt werden, dass das schweizerische Heilmittelrecht bindend ist und die Vorschriften der Europäischen Union mitberücksichtigt werden.

\section{Welche Rolle spielt Swissmedic im (europäischen) Ausland? Wie ist Ihrer Meinung nach die Wahrnehmung von Swissmedic ausserhalb der Schweiz?}

Ich besuche oft internationale Kongresse. Swissmedic wird dort als eigenständige, kompetente und anerkannte Behörde wahrgenommen. Klar haben wir einen Sonderstatus, weil wir nicht zur Europäischen Union gehören. Wir pflegen jedoch einen wissenschaftlichen Austausch mit anderen Behörden. Seit Anfang Jahr tauschen wir auch Daten mit den Heilmittelbehörden von Kanada, Singapur und Australien aus. Das gibt uns international mehr Gewicht und Know-how.

\section{Wie sehen Sie die Zukunft der Komplementärmedizin in der Schweiz?}

Ich sehe ein grosses Potenzial. In Mitteleuropa sind bisher nur etwa 300 Arzneipflanzen gut auf ihre Wirkungen untersucht worden. Das ist weniger als ein Promille aller Pflanzen. Auch werden bisher kaum Heilpflanzen kombiniert verabreicht. Ich hoffe, dass die Anzahl der KPA in Zukunft vergrössert wird, aber auch der bestehende Heilmittelschatz gewahrt bleibt.

\section{Herr Dr. Ziak, haben Sie vielen Dank für das Interview!}

Interview: Alexander Eitner 